\title{
DERMATOLOGICAL IMAGE DENOISING USING ADAPTIVE HENLM METHOD
}

\author{
A. Dogvanich ${ }^{1}$, N. Mamaev ${ }^{1}$, A. Krylov ${ }^{1}$, N. Makhneva ${ }^{2}$ \\ ${ }^{1}$ Faculty of Computational Mathematics and Cybernetics, Lomonosov Moscow State University, Moscow, Russia \\ 119991, Russia, Moscow, Leninskie Gory, MSU BMK \\ a.dovganich@yandex.ru; mamaev.nikolay93@mail.ru; kryl@cs.msu.ru \\ ${ }^{2}$ Moscow Regional clinic of dermatology and venereology, Moscow, Russia
}

\section{Commission II,WG II/10}

KEY WORDS: image denoising, dermatology, optimal denoising parameters, no-reference image quality metrics, mutual information

\begin{abstract}
:
In this paper we propose automatic image denoising method based on Hermite functions (HeNLM). It is an extension of non-local means (NLM) algorithm. Differences between small image blocks (patches) are replaced by differences between feature vectors thus reducing computational complexity. The features are calculated in coordinate system connected with image gradient and are invariant to patch rotation. HeNLM method depends on the parameter that controls filtering strength. To chose automatically this parameter we use a no-reference denoising quality assessment method. It is based on Hessian matrix analysis. We compare the proposed method with full-reference methods using PSNR metrics, SSIM metrics, and its modifications MSSIM and CMSC. Image databases TID, DRIVE, BSD, and a set of dermatological immunofluorescence microscopy images were used for the tests. It was found that more perceptual CMSC and MSSIM metrics give worse correspondence than SSIM and PSNR to the results of information preservation by the non-reference image denoising.
\end{abstract}

\section{INTRODUCTION}

Use of the self-similarity is one of the main classical ideas in image denoising methods. Even CNN based image denoising methods benefit from its use (see (Cruz et al., 2018) as an example). The most known of the self-similarity based denoising algorithms is non-local means (NLM) (Buades et al., 2005). Its weights depend on Euclidean distance between whole blocks (patches) around respective pixels. NLM provides a high quality of the resulting image. However, it has high computational complexity. To overcome this shortcoming of NLM several methods has been proposed including LJNLM-LR (Manzanera, 2010) and GFNLM (Wang et al., 2012). In these methods weights depend on the Euclidean distance between the feature vectors which characterize the patches. In LJNLM-LR components of the feature vector are values of Taylor series expansion coefficients, which, in turn, are the values of image convolution with derivatives of the Gaussian function. One of advantages of (Manzanera, 2010) is the invariance of features to rotation. In GFNLM features are based on Gabor functions. Another shortcoming of NLM is that the method does not consider rotation of blocks i.e. pixels lying on one edge, but with different gradient directions, will be considered different and have small weights. This can lead to poor noise reduction along edges where the gradient has a different direction in each pixel of the edge This shortcoming is overcomed by LJNLM-LR by rotation of components of the feature vector to the coordinate system aligned by the image gradient. We suggest a modification of NLM method using the Hermite function (HeNLM). Components of the feature vector are values of convolutions of source noisy image with multiscale Hermite functions.

Image denoising methods depend on filtering strength parameters. If the source image without noise (or, reference image) is known then an optimal denoising parameter can be chosen by optimization of some metric (for example, PSNR) between filtered image and the reference image. The quality of optimization depends on the selected metrics. The choice of metrics is not trivial task. There is no one opinion how to calculate similarity or difference between two images. In this work we use two most popular quality measures PSNR and SSIM and consider their perseption based extensions MSSIM and CMSC. First is constructed using separate components of SSIM and on an assignment of a physically valid weight function is to each component. Second metric aims to inherit advantages of the both measures.

Nevertheless in practice a reference image is unavailable so a method that works without it (no-reference methods) is needed. One way is to use image quality assessment algorithms to estimate denoising quality. These methods usually calculate image statistics in spatial (Mittal et al., 2012, Moorthy , Bovik, 2011), or frequency (Saad et al., 2012) domains. However, these methods do not take into account the condition of image structure preserving. To control this structures by ridge based approach we calculate image denoising quality using difference between noisy and filtered images (so called method noise (Buades et al., 2005)). A method for automatic parameter selection for image denoising algorithms has been proposed in (Zhu , Milanfar, 2010). It uses structure tensor analysis with fixed scale derivatives estimation. This doesn't allow us to use multiscale approach for image structure analysis. In this paper we use a method for no-reference image denoising quality assessment to choose parameter of a denoising algorithm. We propose to choose denoising parameter by minimization of mutual information of joint distribution of values in method noise image at ridge points along the ridge (Mamaev et al., 2017). We use Hessian matrix eigenvectors and eigenvalues analysis for ridge detection and direction and size estimation. 


\section{HENLM DENOISING ALGORITHM}

HeNLM denoising algorithm (Mamaev et al., 2013) is a modification of the non-local means (NLM) algorithm (Buades et al., 2005). In NLM the value of denoised image $I_{f}(x, y)$ is a weighted sum of values of the source noisy image $I(x, y)$ :

$$
\begin{array}{r}
I_{f}(x, y)=\frac{1}{w(x, y)} \sum_{\left(x^{\prime}, y^{\prime}\right) \in Q(x, y)} w\left(x, y, x^{\prime}, y^{\prime}\right) I\left(x^{\prime}, y^{\prime}\right), \\
w\left(x, y, x^{\prime}, y^{\prime}\right)=e^{-\frac{\left\|v(x, y)-v\left(x^{\prime}, y^{\prime}\right)\right\|_{2}^{2}}{2 \rho^{2}}} .
\end{array}
$$

Here $Q(x, y)$ is a rectangular neighbourhood of pixel $(x, y)$, $v(x, y)$ is the patch of pixels from image $I$ around pixel $(x, y)$, and $w(x, y)$ is normalizer such that

$$
w(x, y)=\sum_{\left(x^{\prime}, y^{\prime}\right) \in Q(x, y)} w\left(x, y, x^{\prime}, y^{\prime}\right) .
$$

HeNLM algorithm replaces the difference between image patches $v(x, y)$ by the difference between feature vectors. The dimensionality of these vectors is much lower than the dimensionality of image patches so the computational complexity for HeNLM algorithm is lower than for NLM. In HeNLM algorithm feature vector components are found as convolutions with Hermite functions $\psi_{n m}^{\sigma}(x, y)$ :

$$
f_{n m}^{\sigma}(x, y)=I(x, y) * \psi_{n m}^{\sigma}(x, y)
$$

Hermite functions can be defined as (Krylov et al., 2002):

$$
\begin{array}{r}
\psi_{n}(x)=\frac{1}{\sqrt{\sqrt{\pi} 2^{n} n !}} e^{\frac{x^{2}}{2}} \frac{d^{n}\left(e^{-x^{2}}\right)}{d x^{n}} \\
\psi_{n}^{\sigma}(x)=\frac{1}{\sigma} \psi_{n}\left(\frac{x}{\sigma}\right) \\
\psi_{n m}^{\sigma}(x, y)=\psi_{n}^{\sigma}(x) \psi_{m}^{\sigma}(y)
\end{array}
$$

The denominator $\sigma$ in (6) is introduced for the equivalence of the filter responses at different scales. The method is similar to LJNLM-LR (Manzanera, 2010), where feature vector components are the values of convolutions of source image with Gaussian derivatives. Some of Hermite functions and Gaussian function derivatives are shown in Figure 1. It can be seen that the localization areas of Hermite functions and Gaussian derivatives are roughly the same but Hermite functions much better allow to represent peripheral parts of the area and have no strong variance of amplitude (for small orders). Hermite functions form the complete orthogonal system in $L_{2}(-\infty,+\infty)$. This lead to high independence of feature vector components in comparison with features used by LJNLM-LR algorithm.

As in LJNLM-LR algorithm the features $f_{n m}^{\sigma}(x, y)$ are calculated in local coordinate system $(g(x, y), \tau(x, y))$ where $g(x, y)$ is image gradient on scale $\sigma$ in point $(x, y)$ and $\tau(x, y)$ is tangential to $g(x, y)$. This allows the features to be invariant to patch rotation. The feature vector is defined as:

$$
\left\{\tilde{f}_{n m}^{\sigma}(x, y): n+m \leq r, \sigma \in S\right\},
$$

where $\tilde{f}_{n m}^{\sigma}(x, y)$ is the feature in the local coordinate system, $r$ is the maximal order of Hermite function, and $S$ is the set of scales. In our calculations we used $r=4$, and $S=\{1,3\}$.

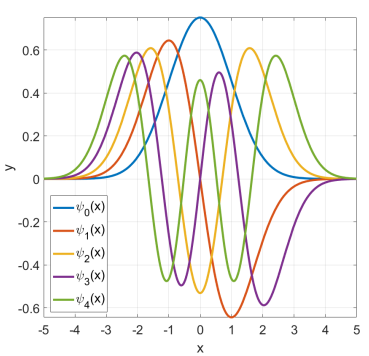

(a)

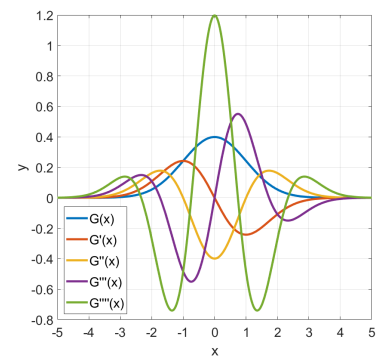

(b)
Figure 1. Hermite functions (a) and Gaussian function derivatives (b)

\section{NO-REFERENCE IMAGE DENOISING QUALITY ASSESSMENT}

We use method noise analysis for image denoising quality assessment. We define method noise image $I_{d}$ for image $I$ as the difference between image $I$ and the image after application of a denoising method $I_{f}$ :

$$
I_{d}=I-I_{f}
$$

If a denoising method works ideally, then method noise image will contain only removed noise. If method noise image contains structured details, then these details have been smoothed or wiped out after application of a denoising method. The absence of correlation of pixel values in method noise image can show that the method noise image contains only noise. While the presence of regular structures results in the presence of correlation of nearby pixel values in area where these structures appear. Image ridge smoothing or suppression by denoising algorithm results in presence of regular structures in the method noise image. So we will analyse the the presence of structure on method noise image in ridge areas (Mamaev et al., 2017).

For ridge detection we use Hessian matrix analysis of the following image:

$$
\begin{array}{r}
L^{\sigma}(x, y)=I(x, y) * G_{\sigma}(x, y), \\
G_{\sigma}(x, y)=\frac{1}{2 \pi \sigma^{2}} e^{\frac{x^{2}+y^{2}}{2 \sigma^{2}}}
\end{array}
$$

We note that the differentiation of $L^{\sigma}(x, y)$ is equivalent to convolution of the source image $I(x, i)$ with corresponding Gaussian function derivative. Let $L_{x x}^{\sigma}(x, y), L_{x y}^{\sigma}(x, y)$, $L_{y y}^{\sigma}(x, y)$ be second derivatives of $L^{\sigma}(x, y)$ by the corresponding variables multiplied by $\sigma^{2}$. This multiplication by $\sigma^{2}$ is used for equalization of Laplacian filter response at different scales (Lindeberg, 1998). Modulus of Laplacian $\Delta L^{\sigma}(x, y)$ has maximal response in the central point of ridge of $2 \sigma$ width. Consider a Hessian matrix:

$$
H^{\sigma}(x, y)=\left(\begin{array}{ll}
L_{x x}^{\sigma}(x, y) & L_{x y}^{\sigma}(x, y) \\
L_{x y}^{\sigma}(x, y) & L_{y y}^{\sigma}(x, y)
\end{array}\right)
$$

The eigenvector $\overrightarrow{v^{\sigma}}(x, y)$ corresponding to the smallest by absolute value eigenvalue of $H(x, y)$ will be directed along the ridge (Lindeberg, 1998). 
Different values of $\sigma \in\left\{\sigma_{1}, \ldots, \sigma_{n}\right\}, \sigma_{i}=\sigma_{0} \cdot \nu^{i-1}$ are used for multiscale ridge detection. Calculating $\Delta L^{\sigma_{i}}(x, y)$ and $\overrightarrow{v^{\sigma_{i}}}(x, y)$, the characteristic ridge size $s(x, y)$ and direction $\vec{v}(x, y)$ are calculated as:

$$
\begin{array}{r}
s(x, y)=\underset{\sigma_{i}}{\arg \max }\left(\left|\Delta L^{\sigma_{i}}(x, y)\right|\right), \\
\\
\vec{v}(x, y)=\overrightarrow{v^{s(x, y)}}(x, y) .
\end{array}
$$

To eliminate false responses of the Laplacian produced by noise for small $\sigma$ we use the following scheme:

1. Apply thresholding to $\left|\Delta L^{s(x, y)}(x, y)\right|$ :

$$
B(x, y)=\left\{\begin{array}{ll}
1, & \left|\Delta L^{s(x, y)}(x, y)\right| \geq T_{l} \\
0, & \text { otherwise }
\end{array} .\right.
$$

We set the threshold value $T_{l}$ to 0.1 in all tests.

2. Find connected components in $B(x, y)$ image and remove components that have less than 5 pixels.

3. Apply binary mask $B(x, y)$ to $\left|\Delta L^{s(x, y)}(x, y)\right|$ to obtain filtered Laplacian responses:

$$
F(x, y)=B(x, y) \cdot\left|\Delta L^{s(x, y)}(x, y)\right| .
$$

To evaluate that correlation we build a joint distribution $p(k, m)$ of the following random variables: $K$ is a value of method noise in point $(x, y)$ and $M$ is a value of method noise in point $(x, y)+s(x, y) \cdot \vec{v}(x, y)$. We use the points where $F(x, y)>0$ as points belonging to ridges. Also we quantize the method noise image intensities to $N$ levels so the nearby intensities are belong to the same event. Bicubic interpolation is used to find intensity values outside the pixel grid. So $p(k, m)$ is defined as follows:

$$
\begin{gathered}
p(k, m)=\frac{1}{P} \cdot \mid\left\{(x, y):\left\lfloor\frac{I_{d}(x, y) \cdot N}{I_{\max }}\right\rfloor=k,\right. \\
\left.\left.\mid \frac{I_{d}(\widetilde{x}, \widetilde{y}) \cdot N}{I_{\max }}\right\rfloor=m, F(x, y)>0\right\} \mid, \\
\widetilde{x}=x+s(x, y) \cdot \vec{v}(x, y)_{x}, \\
\widetilde{y}=y+s(x, y) \cdot \vec{v}(x, y)_{y},
\end{gathered}
$$

where $I_{\max }$ is the maximal allowed pixel intensity value and $P$ is normalizing constant such that $\sum_{k=1}^{N} \sum_{m=1}^{N} p(k, m)=1$. We note that the joint distribution in the case $s(x, y)=s \equiv$ const, $\vec{v}(x, y)=\vec{v} \equiv$ const, and $T_{l}=0$ represents a co-occurrence matrix (Haralick et al., 1973) built for the direction $s \cdot \vec{v}$.

We use mutual information to evaluate independence of random variables $p(k, m)$ :

$$
\mu=\mu(K ; M)=\sum_{k=1}^{N} \sum_{m=1}^{N} p(k, m) \log \left(\frac{p(k, m)}{p(k) p(m)}\right),
$$

where $p(k)$ and $p(m)$ are marginal probability distribution functions:

$$
p(k)=\sum_{m=1}^{N} p(k, m), p(m)=\sum_{k=1}^{N} p(k, m) .
$$

The less is $\mu$ value of the less brightness values of the method noise image along ridges are correlated. We note that the Laplacian $\Delta L^{s(x, y)}(x, y)$ has also a response in the points that belong to edges and blobs. This leads to taking into consideration by the $\mu$ value pairs of pixels belonging to edges and blobs.

The pipeline for computation of $\mu$ value is shown in Algorithm 1. Minimal value of $\mu$ will correspond to the optimal denoising parameter:

$$
\rho_{\text {est }}=\underset{\rho}{\arg \min }(\mu) .
$$

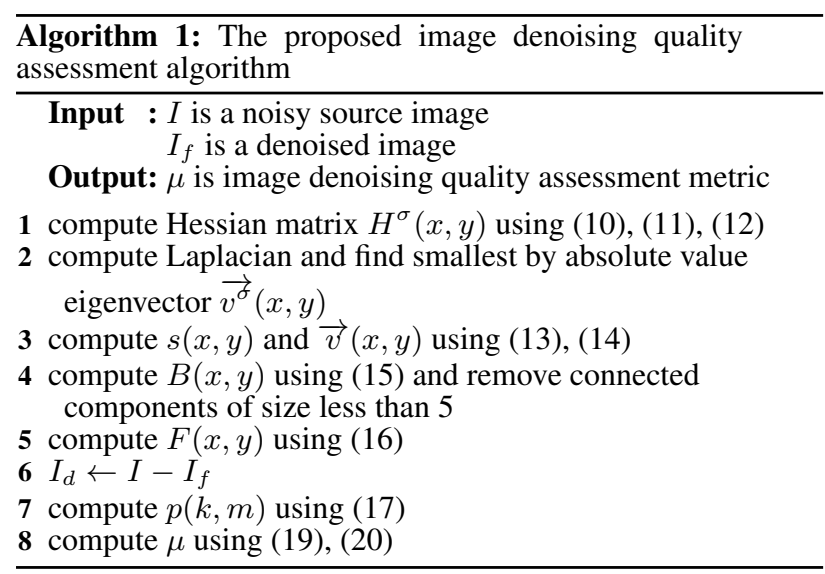

We note that the $\mu$ value will be unstable or even undefined in the case if the Laplacian does not contain enough values larger than $T_{l}$. It also means that the image almost does not contain ridges, edges or other complex structures so edge-preserving denoising is not required. We estimate amount of detected ridges counting the number of points as:

$$
S=|\{(x, y): F(x, y)>0\}| .
$$

If $S$ value is less than threshold $T_{r}=\max (100,0.01 \cdot R \cdot C)$ then denoising is not required and denoising quality assessment is not performed. Here $R$ and $C$ are the number of rows and columns in image respectively. We also note that in the case if there is no noise in source image the $\mu$ value will be monotonically increasing with the increase of the filtering strength since the method noise will not contain noise and denoising should not be performed. To avoid this we check the existence of noise in image using PCA-based noise level estimation method (Pyatykh et al., 2013). If noise level is lower than $\varepsilon$ then denoising is not performed.

\section{FULL-REFERENCE IMAGE QUALITY METRICS}

In this paper we compare the above non-reference denoising parameter estimation method with the full-reference estimation using PSNR, SSIM and modifications of two new SSIM-based similarity measures. First measure is MSSIM (Dovganich et al., 2018) (a modified version of SSIM). The use of MSSIM metrics for the weights estimation gives good results in NLM denoising algorithm. The metrics uses separate components of SSIM and a physically valid weight function is assigned to each component. The standard SSIM contains three components:

$$
\begin{aligned}
& \operatorname{SSIM}\left(x, y, x^{\prime}, y^{\prime}\right)= \\
& l\left(x, y, x^{\prime}, y^{\prime}\right) c\left(x, y, x^{\prime}, y^{\prime}\right) s\left(x, y, x^{\prime} y^{\prime}\right),
\end{aligned}
$$




$$
l\left(x, y, x^{\prime}, y^{\prime}\right)=\frac{2 \mu(x, y) \mu\left(x^{\prime}, y^{\prime}\right)+C_{1}}{\mu^{2}(x, y)+\mu^{2}\left(x^{\prime}, y^{\prime}\right)+C_{1}},
$$

- luminance similarity function,

$$
c\left(x, y, x^{\prime}, y^{\prime}\right)=\frac{2 \sigma(x, y) \sigma\left(x^{\prime}, y^{\prime}\right)+C_{2}}{\sigma^{2}(x, y)+\sigma^{2}\left(x^{\prime}, y^{\prime}\right)+C_{2}},
$$

— contrast similarity function,

$$
s\left(x, y, x^{\prime}, y^{\prime}\right)=\frac{\Gamma\left(x, y, x^{\prime}, y^{\prime}\right)+C_{3}}{2 \sigma(x, y) \sigma\left(x^{\prime}, y^{\prime}\right)+C_{3}},
$$

— structure similarity function.

The constants $C_{1}, C_{2}, C_{3}$ are introduced to prevent division by zero. Here $\mu(x, y)=\langle I(x, y)\rangle$ is image average, $\sigma^{2}(x, y)=\left\langle I^{2}(x, y)\right\rangle-\langle I(x, y)\rangle^{2}$ is image variance, and $\Gamma\left(x, y, x^{\prime}, y^{\prime}\right)=\left\langle I(x, y) I\left(x^{\prime}, y^{\prime}\right)\right\rangle-\langle I(x, y)\rangle\left\langle I\left(x^{\prime}, y^{\prime}\right)\right\rangle$ is image covariance. $C_{1}, C_{2}, C_{3}$ are the stabilization coefficients. As concerned self-similarity based methods like NLM, high weights correspond to blocks that have same structure (pattern) and same brightness and same contrast. Very often fragments of objects in the real world have same structure, but they are observed in various luminance conditions, i.e. have differ brightness and contrast. Moreover, human vision is often not sensitive to such differences and automatically ignores them. Indeed, from the point of view of computer processing, the boundaries of light and shadow are often very important, while human usually ignores them and focuses on the structure of the scene. So, the third multiplier in SSIM can be more important that the first two. Also, and therefore SSIM can take negative values. In theory, it allows to use not only similar patterns, but also their negatives. However, real scenes consisting of positives of some objects and their negatives are very rare in practice. Therefore, we propose to make the third multiplier equal to zero for the case of negative value of the correlation coefficient. We also modified luminosity and contrast multipliers to control the spread of these values. As a result, we get the following expression for MSSIM:

$$
\begin{aligned}
& M S S I M\left(x, y, x^{\prime}, y^{\prime}\right)= \\
& \Theta\left(T_{1} \mu(x, y) \mu\left(x^{\prime}, y^{\prime}\right)-\left(\mu^{2}(x, y)+\mu^{2}\left(x^{\prime}, y^{\prime}\right)\right)\right. \\
& \cdot \Theta\left(T_{2} \sigma\left(x^{\prime}, y^{\prime}\right)-\sigma(x, y)\right) \\
& \cdot \Theta\left(\Gamma\left(x, y, x^{\prime}, y^{\prime}\right)\right) f\left(\Gamma\left(x, y, x^{\prime}, y^{\prime}\right)\right),
\end{aligned}
$$

where $\Theta$ is the Heaviside step function, $T_{1}, T 2$ are the thresholds and we used function $f(x)=x$.

Second measure is CMSC(Palubinskas, 2014). This is a family of metrics that inherits advantages of MSE and SSIM but at the same time avoid their drawbacks. It is based on Means, Standard deviations and Correlation coefficient (CMSC) and consists of the three components: two normalized squared Euclidian measures and one correlation coefficient. Depending on the way of combination three versions are possible. Metrics CMSCam uses averaging and multiplication of individual similarities, CMSCm uses only multiplication of similarities and CMSCa uses only averaging of similarities.

$$
\begin{aligned}
& d_{1}\left(x, y, x^{\prime}, y^{\prime}\right)=\frac{\left(\mu(x, y)-\mu\left(x^{\prime}, y^{\prime}\right)\right)^{2}}{R^{2}}, \\
& d_{2}\left(x, y, x^{\prime}, y^{\prime}\right)=\frac{\left(\left(\sigma(x, y)-\sigma\left(x^{\prime}, y^{\prime}\right)\right)^{2}\right.}{(R / 2)^{2}}, \\
& C M S C_{a m}\left(x, y, x^{\prime}, y^{\prime}\right)= \\
& \left(1-\frac{d_{1}+d_{2}}{2}\right) \cdot s\left(x, y, x^{\prime}, y^{\prime}\right)
\end{aligned}
$$

$$
\begin{aligned}
& C M S C_{m}\left(x, y, x^{\prime}, y^{\prime}\right)= \\
& \left(1-d_{1}\right) \cdot\left(1-d_{2}\right) \cdot s\left(x, y, x^{\prime}, y^{\prime}\right), \\
& C M S C_{a}\left(x, y, x^{\prime}, y^{\prime}\right)= \\
& \frac{2}{3}-\frac{d_{1}+d_{2}}{3}+\frac{s\left(x, y, x^{\prime}, y^{\prime}\right)}{3} .
\end{aligned}
$$

Here $R$ is a normalization constant e.g. $R=255$ for 8 bit data. It is easy to prove that for the normalization of contrast including standard deviations a two times smaller constant $R / 2$ can be used.

\section{COMPARISON METHOD}

For comparison we analyzed the correlation between the considered full-reference similarity metrics parameter estimation results and no-reference mutual information ridge-based technique. For this task we use the following algorithm. We take reference image without noise and add to it Gaussian noise of different levels. After that each image is denoised using HeNLM algorithm and the following extremal problem is solved to find optimal value of the strength parameter $\rho$ using selected full-reference metrics:

$$
\begin{aligned}
& \rho=\underset{\rho}{\arg \max }(f), \\
& f_{\rho}=M\left(I_{\text {ref }}, I_{\text {denoized }}\right)
\end{aligned}
$$

where $M$ is one of the metrics: PSNR, SSIM, MSSIM, CMSC $C_{a m}, C M S C_{m}, C M S C_{a}$.

To find the value of optimal parameter we use maximization algorithm from (Brent, 2013). Finally we compare $\rho$ found by each of the full-reference metrics and the optimal strength parameter found by the no-reference mutual information ridge-based technique using only given noised reference image.

\section{RESULTS}

Image databases TID (Ponomarenko et al., 2015), DRIVE (Staal et al., 2004), and BSD (Martin et al., 2001) were used for the tests. The designed automatic denoising method was also used for dermatological immunofluorescence microscopy images of dermatitis herpetiformis, pemphigus vulgaris and bullous pemphigoid and it showed very good structure preserving denoising results.

Figure 2 shows average values of the absolute difference of values of the denoising parameter automatically found by the proposed mutual information based no-reference method and the values found using full-reference metrics: CMSCa, 
CMSCm, CMSCam, SSIM, MSSIM and PSNR on TID database per noise level. As a result, we can see that the parameter selected using SSIM is the closest to the parameter found by the non-reference method. The average difference over the entire base and for all noise levels is 0.143 for SSIM, 0.1447 for PSNR. Other metrics give worse results with the average value 0.17 . It can be also seen that results for these metrics are less stable than for PSNR and SSIM, but it is mainly caused by the small volume if the TID database. Very close results were obtained for DRIVE database.

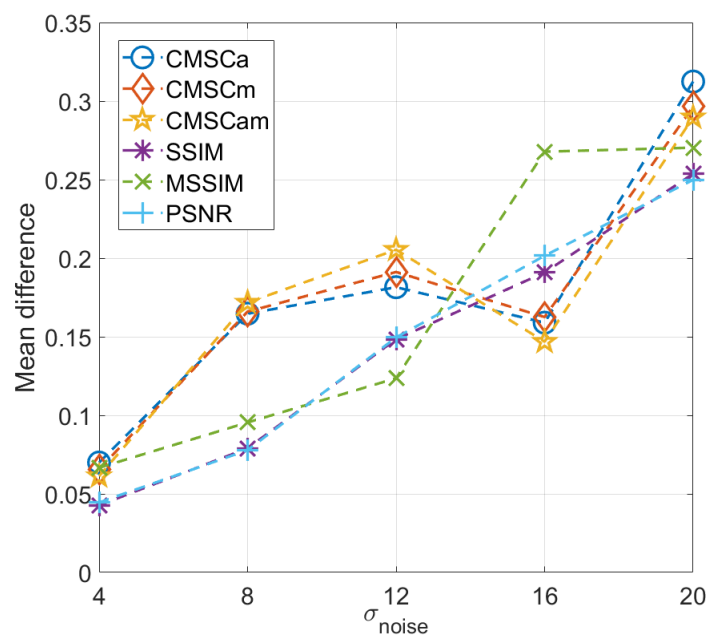

Figure 2. Average values of the absolute difference of parameters found by no-reference mutual information metrics and by full-reference metrics on TID database

The results were also obtained for a larger test database BSDS500 with 500 images. On this database the average difference in parameter for PSNR is 0.1404 , for SSIM is 0.1421 , for CMSCa is 0.1595 , for CMSCm is 0.1559 , CMSCam is 0.1585 , and for MSSIM is 0.1694 . We can see that the dependency graph has become smoother in figure 3 .

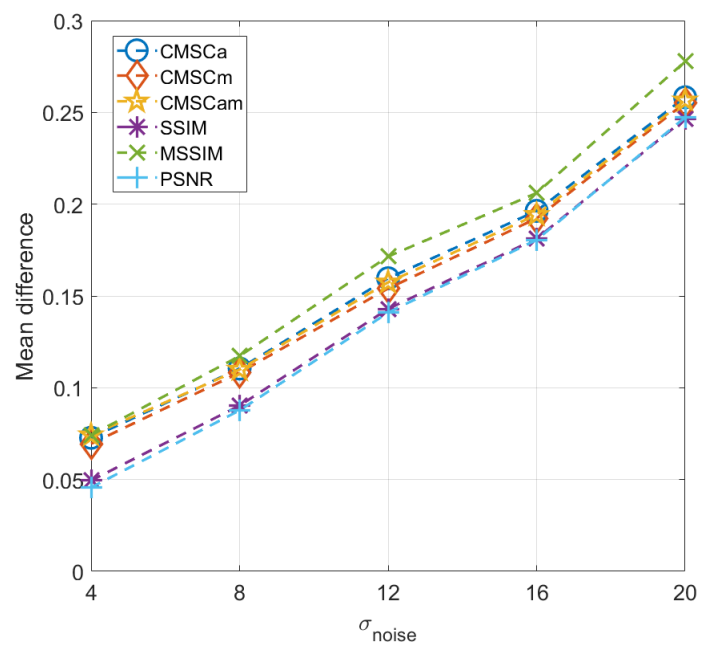

Figure 3. Average values of the absolute difference of parameters found by no-reference mutual information metrics and by full-reference metrics on BSD database

A result of dermatological immunofluorescence microscopy images denoising is shown in figure 4 . The results with the optimal denoising parameter, underestimated parameter (the parameter is 0.5 times less than the optimal) and overestimated parameter (the parameter is 2 times higher than the optimal) are shown.

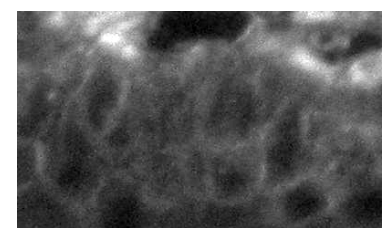

(a)

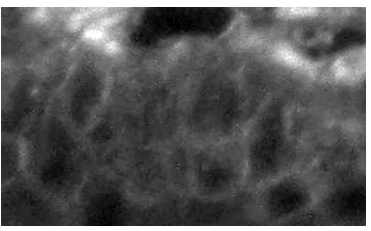

(c)

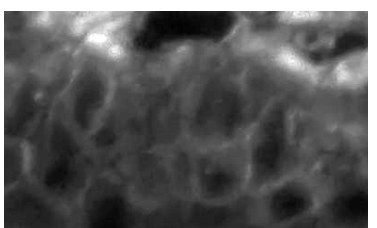

(b)

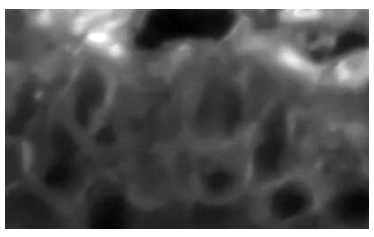

(d)
Figure 4. Results of a pemphigus vulgaris microscopy image denoising. (a) original image, (b) denoising result with optimal parameter, (c) denoising result with low parameter, (d) denoising result with high parameter

A very good correspondence of the obtained optimal denoising parameters to the choice of dermatologists was also found for all examined dermatological immunofluorescence microscopy images.

Test results show that the choice of the metrics that are closer to human vision(CMSC, MSSIM) saves image information after denoising process worse than the choice of PSNR and SSIM. On the other hand when we are using modern metrics we can remove more noise. The price of this often is removing the details unimportant to the human eye, but image becomes more pleasant to see. Correspondingly, if we work with medical images, where every detail can be important, then for the denoising parameter selection is better to use standard metrics (PSNR and SSIM).

\section{CONCLUSIONS}

In this paper we have analysed correspondence of different image metrics to the mutual information based criterion of information preservation after image denoising. It was found that more perceptual CMSC and MSSIM metrics give worse correspondence than SSIM and PSNR to the results of information preservation by the non-reference image denoising.

\section{ACKNOWLEDGEMENTS}

The work was supported by Russian Science Foundation grant 17-11-01279.

\section{REFERENCES}

Brent, Richard P, 2013. Algorithms for minimization without derivatives. Courier Corporation. 
Buades, A., Coll, B., Morel, J. M., 2005. A review of image denoising algorithms, with a new one. SIMUL, 4, 490-530.

Cruz, Cristóvão, Foi, Alessandro, Katkovnik, Vladimir, Egiazarian, Karen, 2018. Nonlocality-reinforced convolutional neural networks for image denoising. IEEE Signal Processing Letters, 25, 1216-1220.

Dovganich, A. A., S., Krylov A., V., Yurin D., 2018. Non-local means algorithm based on modified ssim metrics. GraphiCon 2018: proceedings of 28-th International conference on computer graphics and computer vision, Tomsk Politechnical university, 254-258.

Haralick, Robert M, Shanmugam, Karthikeyan et al., 1973. Textural features for image classification. IEEE Transactions on systems, man, and cybernetics, 610-621.

Krylov, A. S., Kutovoi, A. V., Leow, Wee Kheng, 2002. Texture parameterization with Hermite functions. 12th International Conference Graphicon'2002, Russia, Nizhny Novgorod, 190-194.

Lindeberg, Tony, 1998. Edge detection and ridge detection with automatic scale selection. International Journal of Computer Vision, 30, 117-156.

Mamaev, N., Lukin, A., Yurin, D., Glazkova, M., Sinitsin, V., 2013. Hermite functions expansion based non-local means algorithm for CT applications. 11-th International Conference Pattern Recognition and Image Analysis: New Information Technologies, 2, Russia, Samara, 638-641.

Mamaev, Nikolay, Yurin, Dmitry, Krylov, Andrey, 2017. Image ridge denoising using no-reference metric. International Conference on Advanced Concepts for Intelligent Vision Systems, Springer, 591-601.

Manzanera, Antoine, 2010. Local jet based similarity for NL-means filtering. Pattern Recognition (ICPR), 2010 20th International Conference on, IEEE, 2668-2671.

Martin, David, Fowlkes, Charless, Tal, Doron, Malik, Jitendra et al., 2001. A database of human segmented natural images and its application to evaluating segmentation algorithms and measuring ecological statistics. Iccv, Vancouver.

Mittal, Anish, Moorthy, Anush Krishna, Bovik, Alan Conrad, 2012. No-reference image quality assessment in the spatial domain. IEEE Transactions on Image Processing, 21, 4695-4708.

Moorthy, Anush Krishna, Bovik, Alan Conrad, 2011. Blind image quality assessment: From natural scene statistics to perceptual quality. IEEE transactions on Image Processing, 20, 3350-3364.

Palubinskas, Gintautas, 2014. Mystery behind similarity measures MSE and SSIM. 2014 IEEE International Conference on Image Processing (ICIP), IEEE, 575-579.

Ponomarenko, Nikolay, Jin, Lina, Ieremeiev, Oleg, Lukin, Vladimir, Egiazarian, Karen, Astola, Jaakko, Vozel, Benoit, Chehdi, Kacem, Carli, Marco, Battisti, Federica et al., 2015. Image database TID2013: Peculiarities, results and perspectives. Signal Processing: Image Communication, 30, 57-77.
Pyatykh, Stanislav, Hesser, Jürgen, Zheng, Lei, 2013. Image noise level estimation by principal component analysis. IEEE Transactions on Image Processing, 22, 687-699.

Saad, Michele A, Bovik, Alan C, Charrier, Christophe, 2012. Blind image quality assessment: A natural scene statistics approach in the DCT domain. IEEE transactions on Image Processing, 21, 3339-3352.

Staal, Joes, Abràmoff, Michael D, Niemeijer, Meindert, Viergever, Max A, Van Ginneken, Bram, 2004. Ridge-based vessel segmentation in color images of the retina. IEEE transactions on medical imaging, 23, 501-509.

Wang, Shanshan, Xia, Yong, Liu, Qiegen, Luo, Jianhua, Zhu, Yuemin, Feng, David Dagan, 2012. Gabor feature based nonlocal means filter for textured image denoising. Journal of Visual Communication and Image Representation, 23, 1008 1018.

Zhu, Xiang, Milanfar, Peyman, 2010. Automatic parameter selection for denoising algorithms using a no-reference measure of image content. IEEE transactions on image processing, 19, $3116-3132$.

Revised April 2019 